\title{
Fixed Partial Denture Failures: A Clinical Survey for Evaluation of the Factors Responsible
}

\author{
${ }^{1}$ Manjula B Chandranaik, ${ }^{2}$ Roopa K Thippanna
}

\section{ABSTRACT}

Aim: Most of the time, the failures are conditions that occur during or after appropriately performed fixed prosthodontics treatment procedures. Reasons for failures in fixed prosthodontics can be divided into biological, mechanical and esthetic factors.

The present study was conducted to assess biological, mechanical, and esthetic failure factors among fixed partial dentures (FPDs).

Materials and methods: A cross-sectional study was conducted among patients who reported to the Department of Prosthodontics, College of Dental Sciences, Davangere, Karnataka, India with complaints about fixed partial denture.

A total of 450 fixed partial denture failures in subjects were assessed. The fixed partial denture was examined for the failure factors (biological, mechanical, and esthetic). The selected subjects underwent a clinical examination and set of a questionnaire about the complaint of the fixed partial denture and further detail clinical examination about the failure factor.

Results: Out of 450 fixed partial denture failures, $33.3 \%$ of it showed the biological failure, $55.1 \%$ showed the mechanical failure and $11.5 \%$ showed esthetic failure. The most frequent reason for failure was mechanical factors followed by biological and esthetic failure factors.

Conclusion: The caries was the most common biological failure factor, the loss of retention was the most common cause of mechanical failure factor and the unacceptable color match was accounted more when compared to other esthetic failure factors.

Clinical significance: By knowing the type of failures, we can plan a proper treatment plan so that the abutment will have a long time prognosis.

Keywords: Biological failure, Esthetic failure, Fixed partial denture failure, Mechanical failure.

How to cite this article: Chandranaik MB, Thippanna RK. Fixed Partial Denture Failures: A Clinical Survey for Evaluation of the Factors Responsible. CODS J Dent 2017;9(2):41-45.

\footnotetext{
${ }^{1}$ Senior Resident, ${ }^{2}$ Professor

${ }^{1}$ Department of Dental, SSIMS and RC, Davangere, Karnataka, India

${ }^{2}$ Department of Prosthodontics, CODS, Davangere, Karnataka, India
}

Cooresponding Author: Roopa K Thippanna, Professor, Department of Prosthodontics, CODS, Davangere, Karnataka, India, e-mail: roopakt25@gmail.com
Source of support: Nil

Conflict of interest: None

\section{INTRODUCTION}

Replacement of missing teeth in partially edentulous arch involves various treatment options like removable, fixed prosthesis, and implants. Fixed prosthodontic treatment can offer exceptional satisfaction for both patient and dentist.

Restoring and replacing of teeth with FPDs represents an important treatment procedure in dental practice, mainly because of the continuing high prevalence of caries and periodontal diseases in the adult and geriatric populations. ${ }^{1}$

Failure to achieve the desired specifications of design for function and esthetics would fail the prosthesis. Most of the time, the failures are conditions that occur during or after performed fixed prosthodontics treatment procedures.

Failure of the fixed prosthesis can occur in many ways. The reasons for failure may be divided into biological failures, mechanical failures, and esthetic failures. Mechanical failures are more directly under the influence of the clinician. Biological problems are less easily controlled and in some instances may be unrelated to the treatment or prosthesis. ${ }^{2}$

Reasons of failure can be caries, uncemented restoration, over-contoured restoration, poor occlusal plane, periodontal disease, periapical involvement, failed post retained crowns, poor esthetics, crown perforation and defective margins of restorations. ${ }^{1,2}$

Knowledge regarding the clinical complications that can occur in fixed prosthodontics enhances the clinician's ability to complete a thorough diagnosis and to develop the most appropriate treatment plan. ${ }^{3}$ It provides realistic expectations to patients and to plan the time intervals needed for post-treatment care. ${ }^{4}$

Hence, the present study intended to assess biological, mechanical, and esthetic failure factors among FPDs.

\section{MATERIALS AND METHODS}

A cross-sectional study was conducted among the patients complaining about FPD. The subjects were selected from the Department of Prosthodontics, College 
of Dental Sciences, Davangere, Karnataka, India. The study protocol was reviewed and approved by the Institutional Review Board of College of Dental Sciences, Davangere, Karnataka, India. A total of 450 FPD failures in subjects who were fulfilling the inclusion criteria of the study were selected. The subjects were requested to fill in the consent form and participate in the study. The subjects were evaluated for biological, esthetic and mechanical factors. Questionnaire and clinical examination were performed personally for each subject.

Every subject was asked to sit in the dental chair in an upright position. All the examinations were carried out by the single operator, standing in front of the subjects. Clinical oral examination was done under good illumination using sterile diagnostic instruments. Intraoral examination proceeded in the area of complaint, the type of material made of and the type of pontic design to evaluate the failure factors, i.e., biological, mechanical and esthetic.

Biological failure factors like caries (Fig. 1), tender on percussion, food lodgement, periapical pathology, mobility in abutment, occlusal problem. Caries can be detected by comprehensive probing of the margins of the prosthesis and tooth surfaces with explorer or use of dental floss interproximally the floss thread will get shredded and also the intraoral periapical radiographs to view interproximal caries. Tapping of the abutment with the probe/mirror end at occlusally or incisally to rule out periapical pathology. If percussion did buccally or lingually to rule out periodontal involvement. Palpation of the gum carried out to rule out sinus opening or pus discharge or pain adjacent to the abutment to rule out periapical pathology. Mobility was examined by applying pressure with the ends of two mirrors in buccolingual direction. Interfering centric and eccentric occlusal problems were ruled out by fremitus test, that is by placing the tip of the index finger on the facial surface of the tooth and asked the patient to tap-tap if vibration

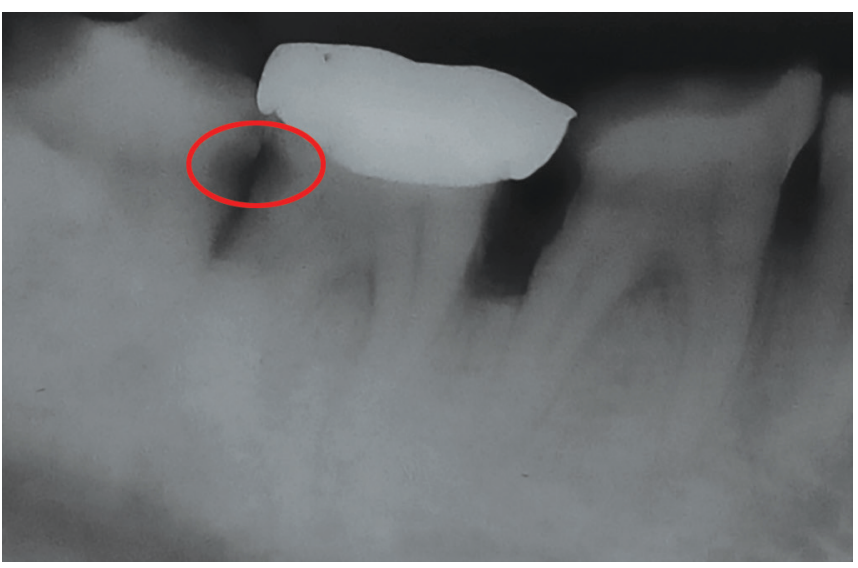

Fig. 1: Biological failure as interproximal caries present then suggestive of occlusal interference or were evaluated by the 100 micron thickness of articulating paper in between the teeth.

Mechanical failure factors like loss of retention, dislodgement of crown or bridge, (Fig. 2), fracture of bridge or connector failure, coronal tooth fracture, occlusal wear of prosthesis or perforation of the prosthesis and porcelain fracture were examined.

Esthetic failure factors (Fig. 3) like unacceptable color match evaluated by comparing with the adjacent teeth or by the patient complaint, over/under the contoured margin of restoration, inadequate marginal fit were examined. The data collected were then subjected to statistical analysis.

\section{RESULTS}

Table 1 shows the frequency distribution of failure factors of the FPD. Out of 450 FPD failures, $33.3 \%$ of it showed the biological failure. $55.1 \%$ showed mechanical failure, and $11.5 \%$ showed esthetic failure. The mechanical failures were more compared to biological and esthetic failures. Table 2 shows the frequency distribution of biological failure factors. Out of 150 biological failure, $30.7 \%$ because

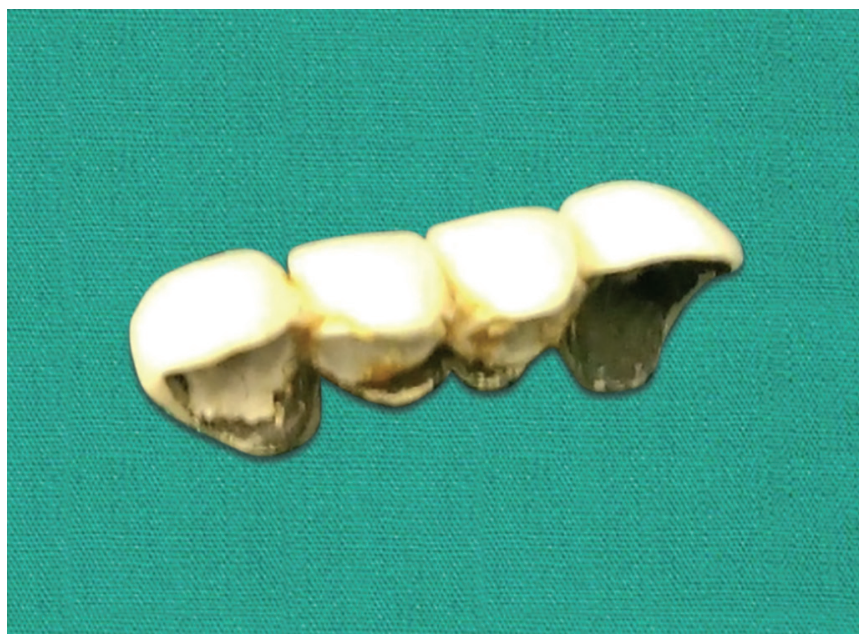

Fig. 2: Mechanical failure as loss of retention

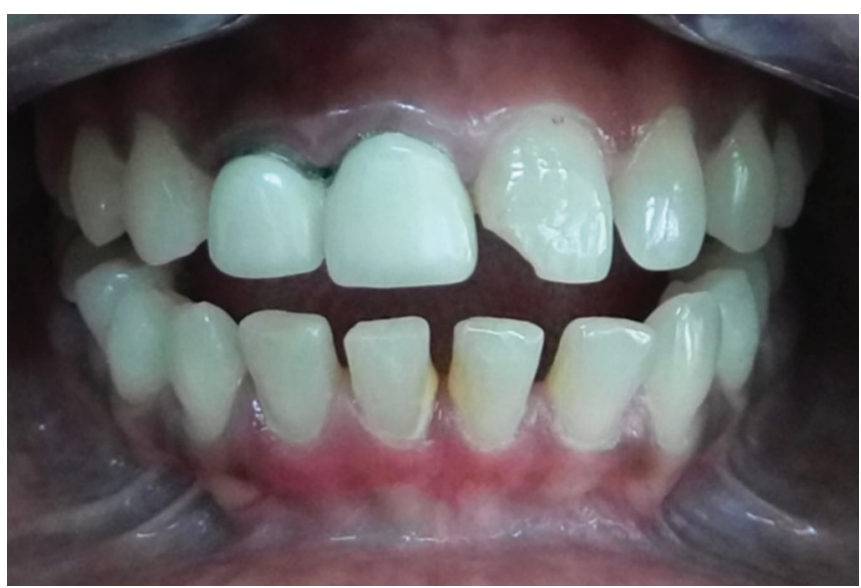

Fig. 3: Esthetic failure as unacceptable color match and under contoured margin 
Fixed Partial Denture Failures: A Clinical Survey for Evaluation of the Factors Responsible

Table 1: Frequency distribution of failure factors of fixed partial denture

\begin{tabular}{lll}
\hline Factors & Frequency & Percentage \\
\hline Biological & 150 & 33.3 \\
Mechanical & 248 & 55.1 \\
Esthetic & 52 & 11.5 \\
\hline Total & 450 & 100 \\
\hline
\end{tabular}

Table.3: Frequency distribution of mechanical failure factor

\begin{tabular}{lll}
\hline Factors & Frequency & Percentage \\
\hline Loss of retention & 164 & 66.1 \\
Fracture of bridge & 8 & 3.22 \\
Coronal tooth fracture & 4 & 1.61 \\
Porcelain fracture & 32 & 12.9 \\
Occlusal wear & 8 & 3.22 \\
$\begin{array}{l}\text { Perforation } \\
\text { Loss of retention due to } \\
\text { fracture }\end{array}$ & 8 & 3.22 \\
$\begin{array}{l}\text { Loss of retention due to } \\
\text { porcelain fracture }\end{array}$ & 8 & 4.83 \\
Loss of retention due to & & \\
perforation & 4 & 3.22 \\
\hline Total & 248 & 1.61 \\
\hline
\end{tabular}

of caries, $18.6 \%$ showed tender on percussion, $24 \%$ showed periapical pathology, 5.3\% showed tenderness on the bite, $8 \%$ showed abutment mobility, $5.3 \%$ of the occlusal problem, $5.3 \%$ food lodgements and $2.7 \%$ showed sinus opening. The caries was the most common biological failure factor, and least was the sinus opening.

Table 3 shows the frequency distribution of mechanical failure factors. Out of 248 of mechanical failures, $66.1 \%$ were because of loss of retention, $3.22 \%$ because of fracture of bridge, $1.61 \%$ coronal tooth fracture, $12.9 \%$ was porcelain fracture, $3.22 \%$ of each occlusal wear and perforation of prosthesis, $4.83 \%$ showed loss of retention due to fracture of bridge, $3.22 \%$ showed loss of retention due to porcelain fracture and $1.61 \%$ showed loss of retention due to perforation of prosthesis. The loss of retention was the most common cause of mechanical failure factor and least was the perforation of the prosthesis and coronal tooth fracture.

Table 4 shows the frequency distribution of esthetic failure factors. Out of 52 esthetic failure, 53.8\% showed unacceptable color match, $7.69 \%$ were of overcontoured margin, and $38.46 \%$ were undercontoured margin. The unacceptable color match was accounted for more when compared to other esthetic failure factors.

\section{DISCUSSION}

With the increased tendency for middle-aged and older persons to retain a relatively high number of their teeth,
Table 2: Frequency distribution of biological failure factors

\begin{tabular}{lll}
\hline Factors & Frequency & Percentage \\
\hline Caries & 46 & 30.7 \\
Tender on percussion & 28 & 18.6 \\
Periapical pathology & 36 & 24 \\
Tenderness on bite & 8 & 5.3 \\
Mobility of abutment & 12 & 8 \\
Occlusal problem & 8 & 5.3 \\
Food lodgement & 8 & 5.3 \\
Sinus opening & 4 & 2.7 \\
\hline Total & 150 & 100.0 \\
\hline
\end{tabular}

Table.4: Frequency distribution of esthetic failure factors

\begin{tabular}{lll}
\hline Esthetic & Frequency & Percentage \\
\hline Unacceptable color match & 28 & 53.8 \\
Overcontoured margin & 4 & 7.69 \\
Undercontoured margin & 20 & 38.46 \\
Total & 52 & 100 \\
\hline
\end{tabular}

various treatment modalities like removable prosthesis, implantology, and conventional bridge prosthesis are possible. If the cost factor precludes the placement of implants, then conventional bridges are still a favorable treatment option as they provide a psychological and social benefit of wearing a fixed rather than removable prosthesis. $^{5-7}$

As the mechanical tolerances are minute and the restorations must survive in a demanding biological environment, the practice of fixed prosthodontics may yield a certain percentage of unsatisfactory outcome which results in failures. ${ }^{2}$

Failure of the fixed prosthesis can occur in due course due to various reasons which may be divided into biological failures, mechanical failures as well as esthetic failures. ${ }^{1,2}$

Hence, it is important to find the causative factors which will help in the management of FPD failures. The condition of failure is identified early and further complication can be prevented so that the prognosis for the FPD is good. So, the study was carried out to assess and evaluate the factors responsible for the failure of FPDs.

The present study was conducted among the individuals complaining about FPD. The subjects were selected from the Department of Prosthodontics, College of Dental Sciences, Davangere, Karnataka, India. A total of 450 FPD failures in subjects were selected. The subjects requested to fill in the consent form and participate in the study. The subjects were evaluated for failure factors namely biological, esthetic and mechanical. 
Data were obtained and subjected to statistical analysis. In the present study, Graph 1 shows the comparison between three types of failures in which biological failures accounted for $33.3 \%$, mechanical failures accounted for the largest number of failures, i.e., $55.1 \%$ and least for the esthetic failures, i.e., $11.5 \%$. The study conducted by Walton et al. on failure factors where mechanical problems accounted more compared to biological and esthetic failures.

Graph 2 represented the distribution of the biological failure factors in which caries was accounted for as the most common cause of failure (30.7\%). Caries had been a major reason for the failure of fixed bridge prostheses according to the studies conducted by different authors. ${ }^{7-12}$ Caries would occur because of poor marginal fit of prosthesis, marginal leakage, high caries index mouth and also because of poor oral hygiene maintenance.

Graph 3 shows the distribution of mechanical failure factors. Loss of retention was the most commonly occurring mechanical failure factor accounted for about $66.1 \%$.

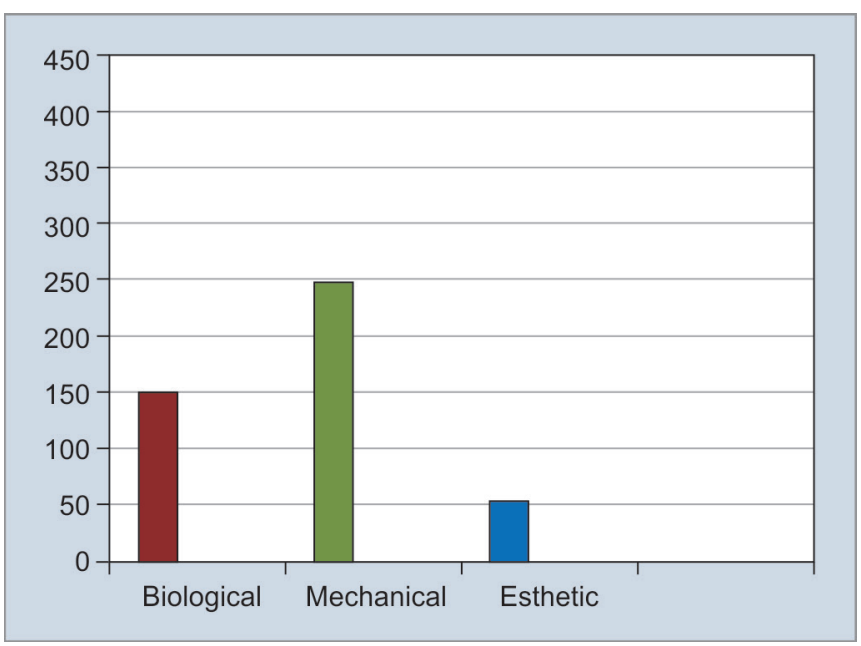

Graph 1: Distribution of failure factors of fixed partial denture

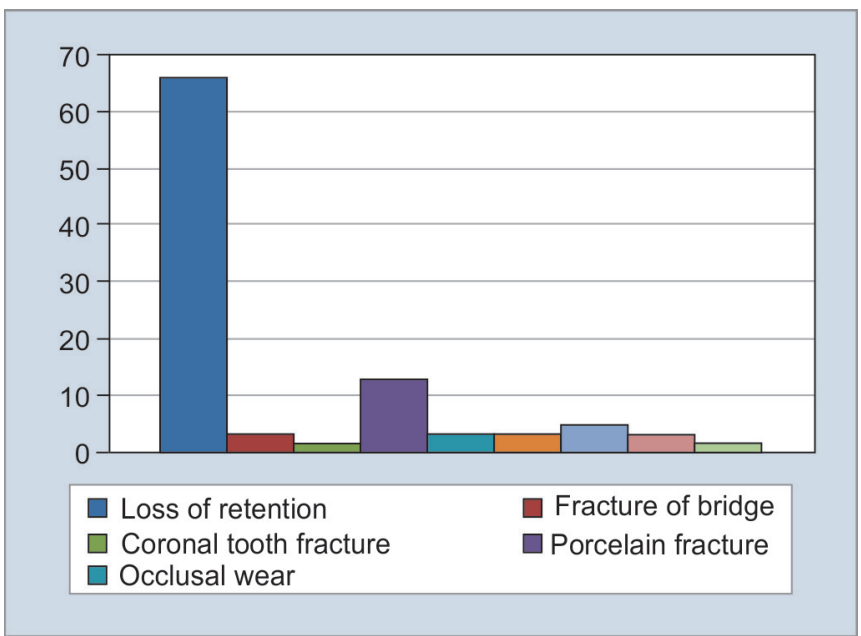

Graph 3: Distribution of mechanical failure factors
Loss of retention means the prosthesis can become loose from an abutment tooth..$^{10}$ The studies conducted by Cheung, ${ }^{13}$ Karlsson in which the loss of retention was the primary cause of failure. The study conducted by Valderhaug shows similar results. The mechanical failure might be due to the cementation failure and excessive taper of abutment tooth resulted in poor resistance form of retainer. ${ }^{10}$

Graph 4 shows the distribution of esthetic failure factors. Among all the esthetic failure factors, unacceptable color match was greatest and was about $53.8 \%$. In the esthetic failure the ceramic restorations more often fail esthetically than mechanically or biologically.

\section{CONCLUSION}

Within the limitations of this study, the following conclusions were drawn:

- The most frequent reason for failure was mechanical failure factors followed by biological and esthetic failure factors.

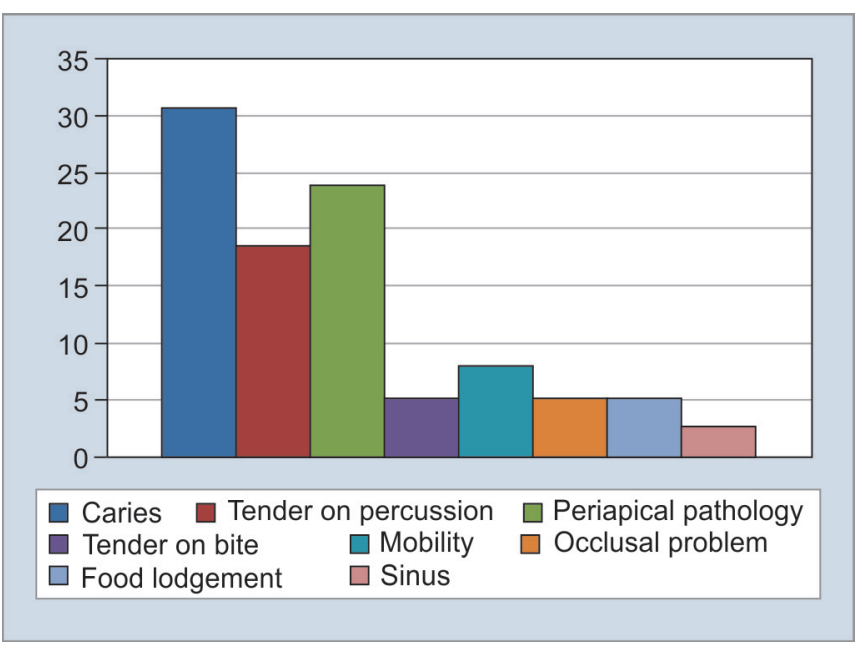

Graph 2: Distribution of biological failure factors

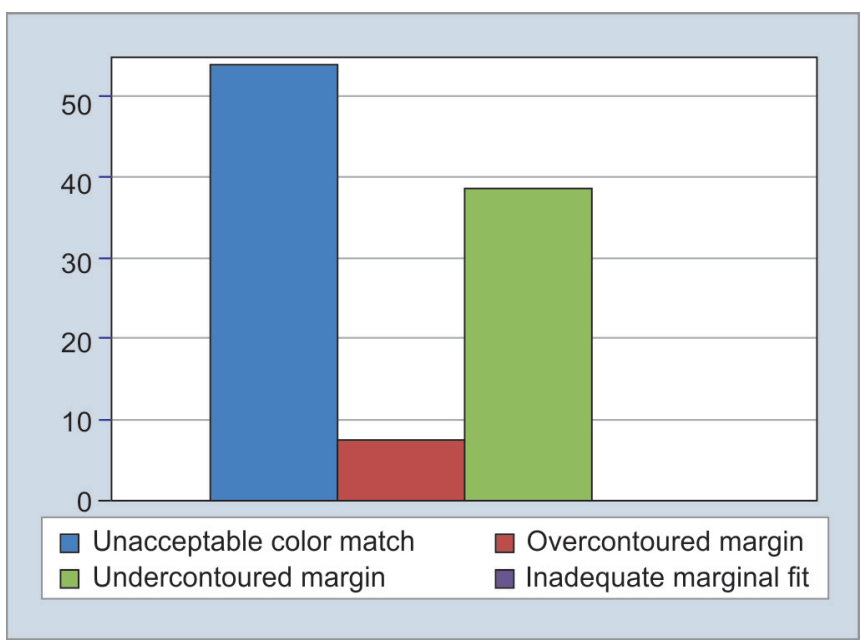

Graph 4: Distribution of esthetic failure factors 
- Loss of retention was the most frequent mechanical factor.

Caries was most frequent biological failure factor.

- Unacceptable color match was the most frequent esthetic failure factor.

\section{REFERENCES}

1. De backer H, Van Maelae G, et al. A 20 year Retrospective survival study of fixed partial dentures. Int J Prosthodont 2006;19:143-153.

2. Selby A. Fixed prosthodontic failure. Aust Dent J 1994;39:150156.

3. Lindquist E ,Karlsson S. Success rate and failures for fixed partial dentures after 20 years of service part I. Int J Prosthodont 1998;11:133-138.

4. Goodacre C J, Bernal G, et al. Clinical complications in fixed prosthodontics. J Prosthet Dent 2003;90:31-41.

5. Näpänkangas R, Salonen-Kemppi MA, et al. Longevity of fixed metal ceramic bridge prostheses: a clinical follow-up study. J Oral Rehab 2002;29(2):140-145.
6. Karlson S. A clinical evaluation of fixed bridges: 10 years following insertion. J Oral Rehab 1986;13:423-432.

7. Randow K, Glantz PO. Technical failures and some related clinical complications in extensive fixed prosthodontics. Acta Odontol Scand 1986;44:241-255.

8. Foster LV. Failed conventional bridgework from general dental practice:clinical aspects and treatment needs of 142 cases. Br Dent J 1990;168:199-201.

9. Glantz POJ, Nilner K, et al. Quality of fixed prosthodontics after 15 years, Acta Odontol Scand 1993;51(4):247-252.

10. Goodacre-Philips D. Text book of Johnston's modern practice in fixed prosthodontics, 4 th edition

11. Reuter JE, Brose MO. Failures in full crown retained dental bridges. Br Dent J 1984;157:61-63.

12. Carlson BR, Yontchev E, et al. Extensive fixed partial dentures on mandibular canine teeth: a 5-year recall study. Int J Prosthodont 1989;2:265-271.

13. Cheung GS, Dimmer A, et al. A clinical evaluation of conventional bridgework. J Oral Rehabil 1990;17:131-136. 